\title{
Acoustic signature of flow instabilities in radial compressors
}

\author{
Elias Sundström ${ }^{1 *}$, Bernhard Semlitsch $^{2}$, Mihai Mihăescu ${ }^{1}$ \\ ${ }^{1}$ KTH - Mechanics, Competence Center for Gas Exchange (CCGEx), Osquars Backe 18, \\ Royal Institute of Technology (KTH), Department of Mechanics, Stockholm, 10044, Sweden. \\ ${ }^{2}$ Whittle Laboratory - Department of Engineering, University of Cambridge, 1 JJ Thomson \\ Avenue. University of Cambridge, Cambridge, CB3 ODY, United Kingdom.
}

\begin{abstract}
Rotating stall and surge are flow instabilities contributing to the acoustic noise generated in centrifugal compressors at low mass flow rates. Their acoustic generation mechanisms are exposed employing compressible Large Eddy Simulations (LES). The LES data are used for calculating the dominant acoustic sources emerging at low mass flow rates. They give the inhomogeneous character of the Ffowcs Williams and Hawkings (FW-H) wave equation. The blade loading term associated with the unsteady pressure loads developed on solid surfaces (dipole in character) is found to be the major contributor to the aerodynamically generated noise at low mass flow rates. The acoustic source due to the velocity variations and compressibility effects (quadrupole in character) as well as the acoustic source caused by the displacement of the fluid due to the accelerations of the solid surfaces (monopole in character) were found to be not as dominant. We show that the acoustic source associated with surge is generated by the pressure oscillation, which is governed by the tip leakage flow. The vortical structures of rotating stall are interacting with the impeller. These manipulate the flow incidence angles and cause thereby unsteady blade loading towards the discharge. A low-pressure sink between 4 and 6 o'clock
\end{abstract}

\footnotetext{
* Corresponding author

Email addresses: elias@mech.kth.se (Elias Sundström ${ }^{1 *}$ ), bs564@cam.ac.uk (Bernhard Semlitsch ${ }^{2}$ ), mihai@mech.kth.se (Mihai Mihăescu ${ }^{1}$ )
}

Preprint submitted to Journal of ${ }^{A} T_{E} X$ Templates

June 13, 2018 
causes a halving of the perturbation frequencies at low mass flow rates operating conditions. From two point space-time cross correlation analysis based on circumferential velocity in the diffuser it was found that the rotating stall cell propagation speed increases locally in the low pressure zone under the volute tongue. It was also found that rotating stall can coexist with surge operating condition, but the feature is then seen to operate over a broader frequency interval.

Keywords: Large Eddy Simulations, Rotating stall, surge, acoustics source, centrifugal compressor

2017 MSC: 00-01, 99-00

\section{Introduction}

Downsized reciprocating internal combustion engines (ICE) in combination with turbocharging play an important role increasing the energetic efficiency and reducing emission levels. In the wide range of engine operating conditions, 5 the turbocharger compressor can become a very important noise contributor [1]. This audible discomfort is caused by the acoustic pressure fluctuations, with the origins in the unsteady pressure loads, some of which driven by the developed flow instabilities interacting with the moving or stationary solid surfaces in the compressor [2, 3].

Rotating stall and surge instabilities may occur during normal driving conditions [4, e.g. gear shifting or sudden acceleration. Experimental measurements by Kabral and Åbom [5] provides scientific evidence that these off-design flow instabilities cause substantial sound pressure levels.

Without a complete description of the temporal and spatial evolution of the flow instabilities and the unsteady pressure loads on solid surfaces there is a lack of knowledge regarding the sound generation mechanism. This restricts the optimization of compressors with respect to acoustic performance [6, 7]. Rämmal and Åbom [8] reported that such sources can be categorized according to their characteristics and are commonly referred to as monopoles, dipoles, 
and quadrupoles. A monopole source is associated with temporal variations caused by motion of a discontinuity forcing a net volume flow (e.g. impeller blade surface). A dipole source corresponds to pressure fluctuations on a solid surface induced by e.g. flow structure interaction with the surface or flow separation. Quadrupole sources originate from turbulent fluctuations or by varying tangential shear stresses on surfaces.

Several distinct acoustic noise features have been reported in the literature. However, a quantification of the acoustic sources has not been carried out. A distinct mode is associated with the blade passing frequency (BPF), i.e. the rotating order times the number of impeller blades. Narrowbanded tip clearance noise has been reported by [9] to dominate over the blade passing tones. It has been reported to occur at approximately $50 \%$ of the rotating order frequency (RO) [10] for some compressor designs and may be more prominent at lower impeller speeds. Additionally, it has been suggested to emanate from secondary flow motion in the gap between the compressor casing and the impeller blades similar to axial compressors. Moreover [10 hypothesized for an existence of a relationship between rotating flow instability and tip clearance noise. However, in the work by 11 the tip clearance size is reported to have negligible effect on the noise generation at off-design operating conditions. Mendonca et al. [12 found that the occurrence of stalled impeller blades is related to a rotating instability 40 and with associated amplified noise levels. The presence of narrowbanded noise at higher harmonics to the shaft rotational speed has been reported by 13 . Another noise source, evident at near-surge line operating conditions, is coined "whoosh noise" [14, 15, stretching over several orders of kHz. It was observed that the "whoosh noise" is more apparent at near-surge operating conditions than at actual deep-surge operating conditions, see [14, 2].

Karim et al. [16] related high flow incident angles at the leading edges of the impeller with noise generation and suggested that rotating flow structures in the blade passages are causing the so called "whoosh noise". In contrast, [14] observed the main noise source to be localized further downstream in the 50 compressor outlet piping. All these studies reveal the challenge associated with 
connecting the perceived acoustic noise with the actual generation mechanism. One of the best-known descriptions for noise assessment of rotating devices such as impellers is the Gutin analogy [17. An alternative theory is according to [18. Both models have in common that the net force produced by each blade is assumed constant. The resulting force distribution yields a source signal with a dipole character. It consists of the fundamental blade passing frequency including higher harmonics. With simplifying assumptions such as compact source field, the analogy suggests a high directionality of the far-field sound, i.e. zero intensity perpendicular and co-planar to the impeller disc. From observations, this never happens, because effects from unsteady flow disturbances will tend to manifest in a broadband spectrum. Therefore, a deterministic acoustic model is not completely satisfactory to describe the emitted noise for centrifugal compressors. An obvious shortcoming is the inability of the reduced order models to account for sound associated with low-frequency tonalities. In fact most of the audible frequency range is unaddressed. More predictive capabilities were demonstrated in [1] using unsteady Detached Eddy Simulation (DES). The computed pressure fluctuation spectra were reported to show good trends with experimental data.

The current work is looking to reveal the interconnectivity between the flow field instabilities, the acoustic sources, and the observed emitted sound levels. High-fidelity compressible Large Eddy Simulation (LES) calculations are employed in order to quantify the developing flow instabilities as the compressor operating conditions approach the surge line. This methodology, i.e. LES has also demonstrated predictive quality for a range of operating conditions as compared to probe point spectra obtained experimentally, see [19, 20. Acoustic sources are determined using the Ffowcs Williams-Hawkings (FW-H) equation 21]. The distribution of the acoustic source signatures are extracted using modal decomposition methods. Further, the acoustic sources are analyzed and linked with the generating flow structures. From the theoretical stand point of view Howe 22] derive that radiated power due to pulsating pipe flow is propor- 
tional to:

$$
\frac{\bar{W}_{m}}{\rho_{0} U^{3} D^{2}} \propto M
$$

where $\rho_{0}$ - air density, $U$ - mean flow speed, $D$ - pipe diameter, $c_{0}$ - sound speed, $M$ - Mach number, and subscript $m$ refers to a monopole source. The expression in Eq. 1 is obtained by assuming that the frequency of the monopole source is proportional to $U / D$. Moreover, the power is normalized with $\rho_{0} U^{3} D^{2}$ so that radiation efficiency is only proportional to the Mach number. The rotating blade forces can be represented as a dipole radiation. The radiation efficiency from a dipole source is also derived in Howe 22] shown to be proportional to the cube of the Mach number:

$$
\frac{\bar{W}_{d}}{\rho_{0} U^{3} D^{2}} \propto M^{3}
$$

where subscript $d$ refers to a dipole. The Lighthill stress tensor behaves as a quadrupole acoustic source. For this Howe derives that the quadrupole radiated power scales as:

$$
\frac{\bar{W}_{q}}{\rho_{0} U^{3} D^{2}} \propto M^{5}
$$

where the quadrupole is indicated with subscript $q$. It needs to be emphasized that Eqs. 1 3 relates only to the effectiveness of the source type with respect to the Mach number. It does not convey the actual magnitude of the source in the present configuration. Therefore, LES together with FW-H can help to complement the theory and establish more fidelity in the acoustic power scaling law for centrifugal compressors.

Compressible Large-Eddy Simulation (LES) calculations are used to quantify the flow and the near-field acoustics associated with a centrifugal compressor at stable and off-design (unstable) operating conditions. Featured instabilities in the flow field at off-design operating conditions are linked with acoustic noise generation.

\section{Geometry and Numerical Methodology}

Side and front views of the investigated centrifugal compressor are presented in Fig. 1. The compressor features a ported shroud supported by four ribs in 
an asymmetric arrangement. An impeller with 10 main blades (exducer diameter $D_{2}=88 \mathrm{~mm}$ ) is considered and the compressor accommodates a vaneless diffuser. A bell mouth is fitted at the inlet and an idealized straight duct is considered at the volute discharge. The experimental data from University of Cincinnati (UC) [23] have been used for verifying and validating the compressible solver employed. A general good agreement between predicted and measured data in terms of time-averaged velocity, static pressure distribution, frequency spectra, and compressor performance parameters (pressure ratio and efficiency) has been found [19, 20, 24].

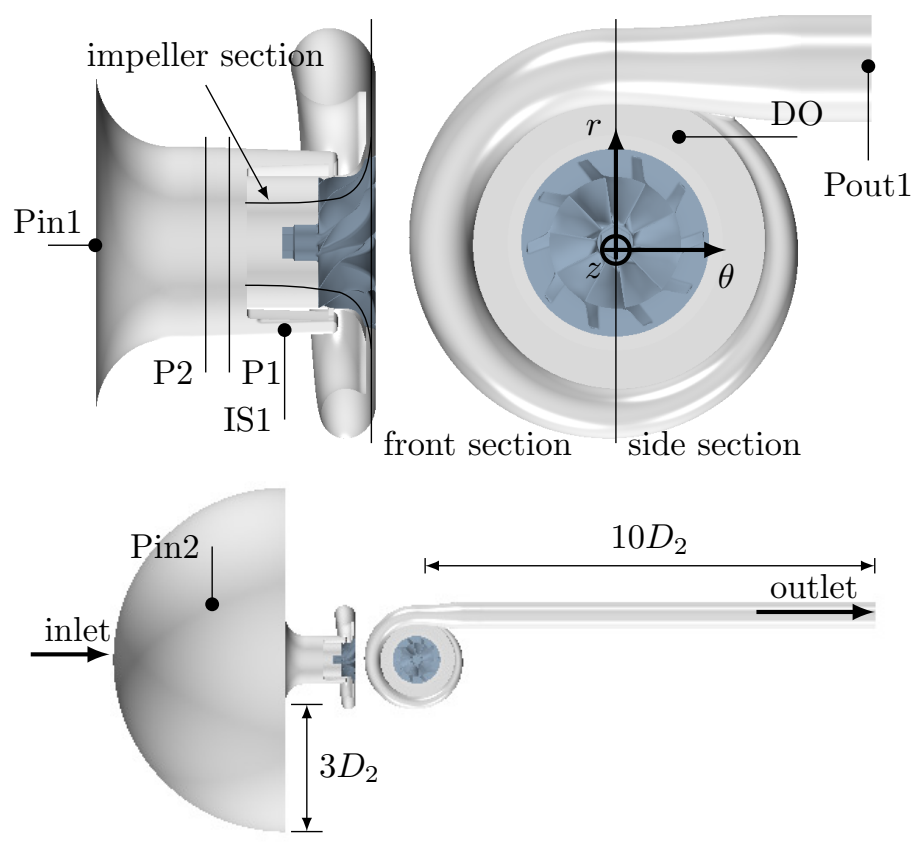

Figure 1: Side and front views of the centrifugal compressor CAD geometry. Locations of the pressure sensors D0, IS1, Pin1, Pin2 and Pout1 are indicated as well as the location of planes used during data post-processing. The impeller section intersects the impeller region at $50 \%$ blade span. The P1 and P2 planes intersect the inlet duct upstream of the impeller eye. The bottom subfigure depicts inlet and outlet boundaries of the computational domain. A larger hemisphere extruded three diffuser diameters is fitted upstream of the bell mouth inlet and the outlet boundary is extruded ten diffuser diameter $2^{2}$ 
Continuity, momentum and energy conservation constitutes the compressible flow governing equations and are solved numerically using the finite-volume compressible solver in STAR-CCM+. A compressible Newtonian fluid is assumed together with constitutive relations to couple velocity gradients to components of the stress tensor. The dynamic viscosity is thermally variant by means of the Sutherland equation. The fluid is assumed approximately calorically perfect with constant specific heats. Molecular diffusion fluxes of heat and mass are assumed to satisfy Fourier's and Fick's laws, respectively. The ideal gas equation of state is used to close the system of equations.

Three different operating conditions are considered at a constant speedline of $64 \mathrm{krpm}$, see Tab. 1. Case D corresponds to a stable condition. For the other cases, the mass flow rate is restricted with purpose to provoke flow instabilities known as rotating stall and surge. The total pressure ratio TPR and the isentropic efficiency $\eta$ were previously evaluated [20] to assess the global performance of the centrifugal compressor stage. These parameters were found to be in good agreement with experimental data 23 .

Table 1: Operating conditions with target mass flow rate and target static pressure at the outlet provided by experimental measurements. The last column indicates the Reynolds number based on the blade tip speed $U_{t i p}$, the exducer radius $R_{2}=44 \mathrm{~mm}$, and the kinematic viscosity of air $\nu$.

\begin{tabular}{lccc}
\hline Case & $\dot{m}(\mathrm{~kg} / \mathrm{s})$ & $p_{\text {exit }}(\mathrm{kPa})$ & $R e_{R_{2}}=\frac{U_{t i p} R_{2}}{\nu}$ \\
\hline A (surge) & 0.050 & 165 & $1.1 \cdot 10^{6}$ \\
B (rotating stall) & 0.085 & 165 & $1.1 \cdot 10^{6}$ \\
D (stable) & 0.280 & 156 & $1.1 \cdot 10^{6}$
\end{tabular}

Upstream of the bell mouth inlet, the fluid is assumed to be approximately quiescent with known quantities of stagnation temperature and pressure. Freestream Mach number, free-stream static temperature, and free-stream pressure

\footnotetext{
${ }^{2}$ The back pressure and thus the mass flow is regulated via a valve in the experiments 23, which has been modeled by a mass flow rate boundary condition in the simulations.
} 
corresponding to the target mass flow rate from Tab. 1 are specified on the inlet boundary. These free-stream values are used together with characteristics extrapolated from the neighboring cell center in order to compute the condition on the boundary face, for more details on this treatment see [25, 26]. The idea with this boundary condition implementation is to adjust and correct the assigned boundary face properties depending on incoming compression waves that may be radiated from sound sources within the compressor. The grid is gradually stretched towards the inlet, which provides a numerical sponge zone. The hemispherical inlet surface is located about three diffuser diameters upstream from the bell mouth entrance to the compressor as shown in Fig. 1 . Adiabatic non-slip walls are considered on all solid surfaces.

The implicit Large Eddy Simulation (LES) approach is considered for resolving the large scale structures in the flow field. The governing equations are cast into continuous integral form and then formulated for discrete finite control volumes that constitute the computational domain. The transient term is discretized with a second-order temporal scheme. Convective flux and diffusive flux terms are discretized with a hybrid third order spatial scheme, which blends between upwind and central formulation. Introduction of discretization schemes cause truncation errors. This error has been demonstrated to be in the same order of magnitude as compared to a Smagorinsky SubGrid Scale (SGS) model, see e.g. [27, 28].

Due to the geometrical complexity an unstructured polyhedral grid is considered consisting of approximately $9 \cdot 10^{6}$ cells. The near wall region is resolved at non-slip walls with prismatic cell layers due to the large wall normal velocity gradient. A low Reynolds-number wall-resolved grid with a wall y+ equal unity on average is achieved with 10 prism layers, a total prism layer height of 0.5 $\mathrm{mm}$, and with a stretch factor of 1.5. For numerical robustness an all wall $\mathrm{y}+$ formulation is therefore employed.

To accommodate well-conditioned boundary conditions the grid is gradually stretched towards the openings of the computational domain. To handle the impeller rotation and capture the flow-impeller interaction the domain is 
divided in a stationary region and a rotating region with a sliding interface in between [29]. The two sides remain implicitly coupled and the cell connectivity is recalculated at each time-step. Fluid information in time and space are interpolated to preserve flux continuity across the interface to avoid introducing spurious perturbations to the flow field. The grid of the non-stationary part is made to rotate at a constant angular velocity with respect to the compressor axis.

The Ffowcs Williams Hawkings (FW-H) analogy enables extrapolation of the sound emitted from a simulated flow scenario to the far-field. The idea is to estimate small pressure fluctuations at receiver locations. The equation for pressure is derived in 21] and is computed as a sum of a monopole term, a dipole term, and a quadrupole term.

$$
p^{\prime}(\bar{x}, t)=p_{T}^{\prime}(\bar{x}, t)+p_{L}^{\prime}(\bar{x}, t)+p_{Q}^{\prime}(\bar{x}, t)
$$

The monopole term is given as follows:

$$
p_{T}^{\prime}(\bar{x}, t)=\frac{1}{4 \pi}\left(\frac{\partial}{\partial t} \int_{S}\left[\frac{Q}{r\left(1-M_{r}\right)}\right]_{r e t} d S\right)
$$

with $Q=\rho_{0} U_{i} n_{i}$ and $U_{i}=\left(1-\frac{\rho}{\rho_{o}}\right) v_{i}+\frac{\rho u_{i}}{\rho_{0}}$ where $u$ - fluid velocity, $v$ - control surface velocity, $\rho_{0}$ - far field density. The dipole term is given by:

$$
p_{L}^{\prime}(\bar{x}, t)=\frac{1}{4 \pi}\left(-\frac{\partial}{\partial x_{i}} \int_{S}\left[\frac{L_{i}}{r\left(1-M_{r}\right)}\right]_{r e t} d S\right)
$$

with $L_{i}=P_{i j} n_{i}+\rho u_{i}\left(u_{n}-v_{n}\right)$ and $P_{i j}=\left(p-p_{0}\right) \delta_{i j}-\sigma_{i j}$. The parameter $u_{n}$ is the fluid velocity component normal to the surface, $n_{i}$ - is the surface normal vector, $\sigma_{i j}$ - is the viscous stress tensor, $P_{i j}$ - is the compressive stress tensor. Finally the quadrupole term is:

$$
p_{Q}^{\prime}(\bar{x}, t)=\frac{1}{4 \pi}\left(\frac{\partial^{2}}{\partial x_{i} \partial x_{j}} \int_{V}\left[\frac{T_{i j}}{r\left(1-M_{r}\right)}\right]_{r e t} d V\right)
$$

where $T_{i j}$ - is the Lighthill stress tensor. The subscript ret is short hand for retarded time, and indicates the time of emission. The parameter $M_{r}$ in the integrand's denominator is the Mach number for the source towards the observer location and where $r_{i}$ is the distance from the source point to the observer. 
Different source estimation formulations of the FW-H model have been proposed suitable for different applications. In this study the Dunn Farassat Padula 1A formulation is used [30, which is customized for rotor blade applications.

The integrated emission surface is assumed to coincide with the impeller surface such that a monopole source evolves due to the thickness surface term and a dipole source arises from the blade loading surface term. The thickness surface term $p_{T}^{\prime}(\bar{x}, t)$ results from fluid displacement due to the impeller rotation. It gives an acoustic contribution due to the displacement of the fluid. The fluid is dislocated by the acceleration of a moving surface of given geometry and thickness. The blade loading surface term $p_{L}^{\prime}(\bar{x}, t)$ represents unsteady transport of the surface force distribution on the impeller. The physical interpretation is an acoustic contribution caused by unsteady fluctuation load forces on the solid surface. All visible surfaces in the enlarged top view in Fig. 1 are defined as FW-H source surface locations of the noise.

The quadrupole term $p_{Q}^{\prime}(\bar{x}, t)$ accounts for turbulent mixing as a volumetric source, which is modeled by a "collapsing-sphere" formulation, as suggested by [31]. For computational efficiency this is cast into the "source-time-dominant" algorithm from 32. In this formulation the time derivatives are shifted inside the source term integrals, which circumvent numerical time differentiation of the integrals. The rotating impeller region is chosen as the FW-H volume, which accounts for nonlinearities. The physical interpretation of the quadrupole term is an acoustic contribution due to velocity variations in the fluid flow.

Statistics for a total of 200 rotor revolutions are computed to converge the spectral averages. Grid sensitivity and validity of result w.r.t. available experimental data has been documented in previous publications, see Sundström et al. 19, 20, 33. The considered numerical setup has been shown to capture at least one order of magnitude of the inertial subrange. It is hence regarded a necessary and a sufficient technique to capture the hydrodynamic flow in the compressor.

A grid dependency study is also performed to assess the validity of the acoustic propagation into the far-field. Therefore, a sine wave is introduced as a 
momentum source, with an amplitude $a_{r e f}=100 \mathrm{~Pa}$ and frequency $\omega_{\text {ref }}$ equal

\section{Results}

Cross-correlations have been carried out between pressure signals acquired at different locations in the compressor. The purpose was to establish correlations between the observed modes inside the compressor (IS1 at the impeller's inlet, D0 in the vaneless diffuser) and those dominant in the upstream hemispherical domain (Pin1 and Pin2 in the near-field acoustic region) or in the outlet pipe (Pout1), respectively. See also Fig. 1 for location of the monitoring points. The 


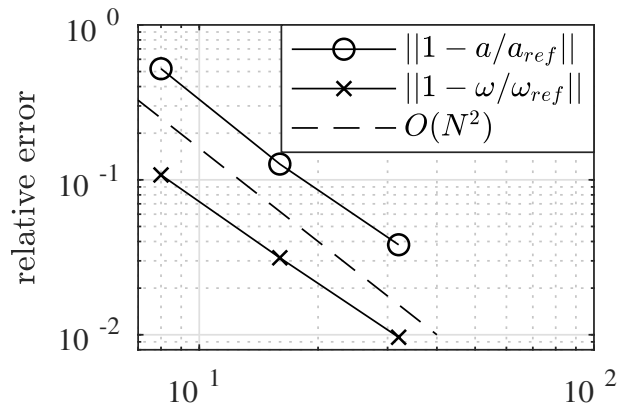

a) $\quad N$

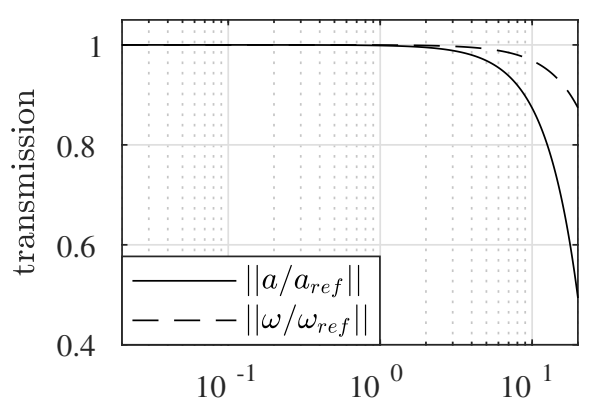

b) $\mathrm{RO}$

Figure 2: Grid dependency assessment of a propagating plane wave. a) Amplitude error, where $a_{\text {ref }}=100 \mathrm{~Pa}$, and frequency error, where $\omega_{\text {ref }}$ is equal the blade passing frequency (BPF). The symbol $N$ depicts the number of grid points per wave length. Both amplitude and frequency errors are seen to reduce two orders of magnitude for each grid refinement order. The amplitude error is approximately one order higher as compared with the frequency error. b) Sensitivity of the amplitude and the frequency errors with respect to the rotating order RO. The error reduction rates are extrapolated from subplot a) for the constant grid resolution $N=2^{4}$.

Cross Power Spectral Densities (CPSD) of the sampled pressure signal between the monitoring points are presented in Fig. 3, exhibiting the distinctive character for different operating conditions. The Rotating Order (RO) represents a non-dimensional frequency, and when it is equal unity it corresponds to the angular velocity of the impeller shaft. A tonality at $\mathrm{RO}=10$ including its higher harmonic is present for all operating conditions. It represents the blade passing frequency (BPF) since the impeller has 10 blades. The CPSD for Cases $\mathrm{A}$ and $\mathrm{B}$ reveal another tonality at $\mathrm{RO}=0.5$, which is not captured for the stable operating Case D. A peak at $\mathrm{RO}=0.04(43 \mathrm{~Hz})$ is visible solely for Case A, with its higher harmonic being present only at some probe locations. Locations deep in the compressor (Fig. 3 (c) and (d)) exhibit a similar broadband CPSD with the aforementioned case-specific tonalities. Further upstream towards the compressor inlet region (Fig. 3 (a) and (b)), the differences between the amplitudes calculated for the unstable scenarios (A and B) and the stable Case D increase, in particular within the low and mid-frequency range. The 
largest amplitudes are found for Case A. One can note also that the spectra in Fig. 3(a) correspond to the near-field acoustic region, which is dominated by acoustic pressure fluctuations.
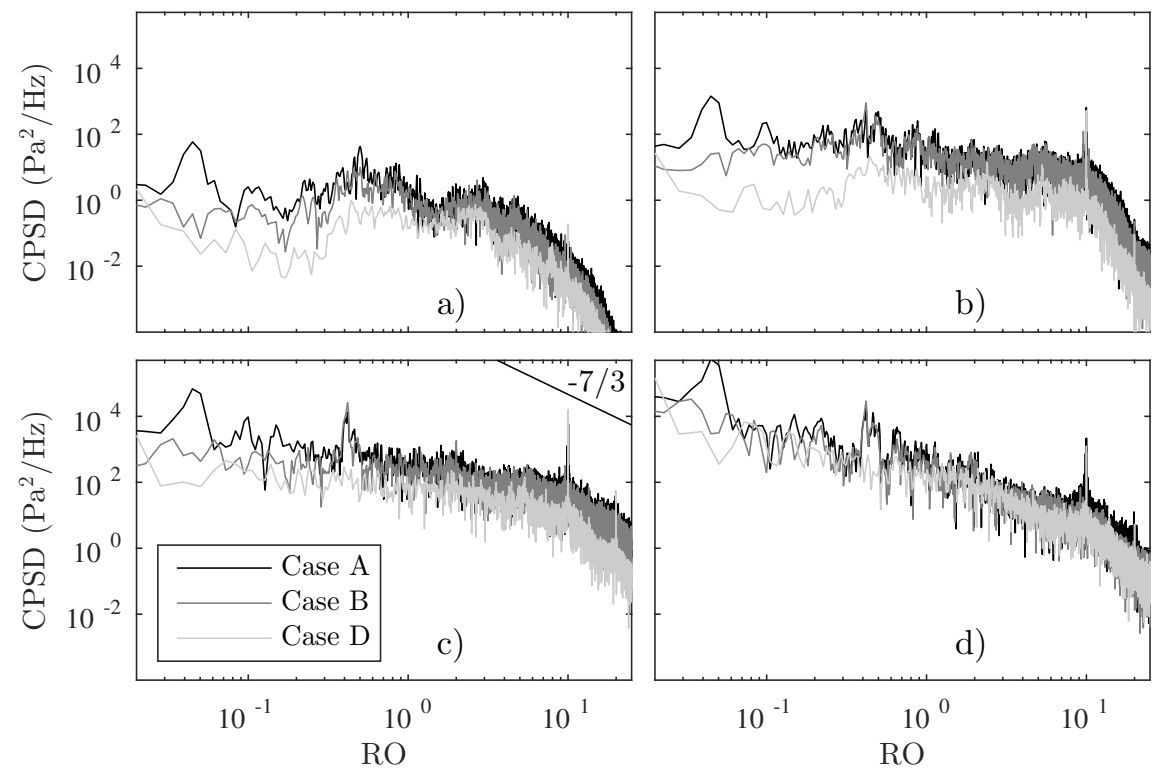

Figure 3: Cross Power Spectral Density between monitoring points for flow Cases A, B and D. a) Pin2 and Pin1. b) Pin1 and IS1. c) IS1 and D0. d) D0 and Pout1. For clarification of monitoring point locations see Fig. 1. All the operating conditions are simulated for the same impeller rotating speed $64000 \mathrm{rpm}$, moving from a higher mass flow rate (Case D, i.e. stable operating condition) towards a lower mass flow rate (Cases B and A).

The CPSD spectra based on the pressure fluctuation data in the entrainment region, upstream of the compressor inlet is depicted in Fig. 3(a). A hump in the low-frequency range with peak amplitude at $\mathrm{RO}=0.5$ is visible. $\mathrm{A}$ second amplification establishes at a higher frequency at approximately $\mathrm{RO}=$ 3. This behavior has been observed for ducted fan configurations, see e.g. 34]. Moreover, the low frequency noise propagating in the upstream direction got amplified by changing the configuration of the duct inlet from a bell-mouth inlet to a rectangular one.

Figure 4 presents the instantaneous hydrodynamic and propagating acoustic 

tra. The acoustic waves traveling upstream form hemispherical waves with circumferential distributed highs and lows due to the interference effects. Pressure fluctuations in the hydrodynamic region of the domain are also distinguishable. These are visible in the shear-layer developed at the bell-mouth inlet towards scale turbulence related fluctuations. These have inherently higher magnitudes than acoustic waves.

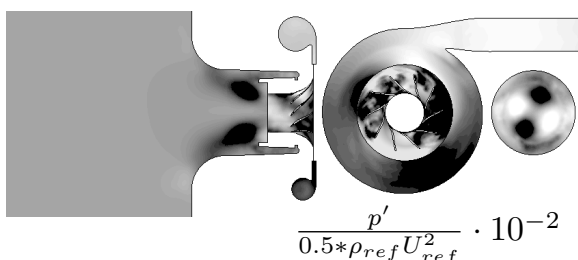

a) $\mathrm{RO}=0.5$
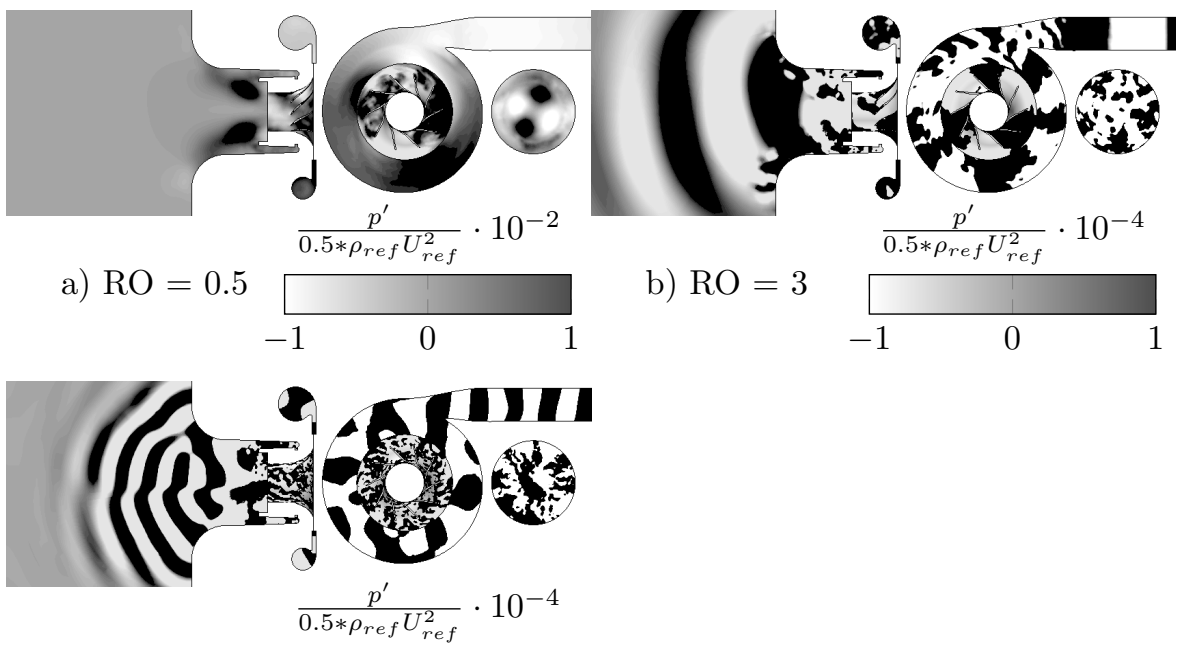

c) $\mathrm{RO}=10$

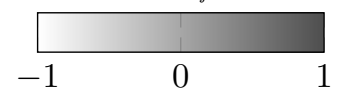

Figure 4: Hydrodynamic and acoustic pressure fluctuations obtained from the solution of the compressible governing flow equations for Case A. Data is sampled at specific frequencies a) $\mathrm{RO}=0.5, \mathrm{~b}) \mathrm{RO}=3$, and c) $\mathrm{RO}=10$. Spherical waves at different wave lengths characteristic to the specific mentioned frequencies are manifested in the upstream far-field and the discharge outlet pipe. Hydrodynamic pressure fluctuations at larger magnitudes are observed inside the compressor. For the location of the post-processing planes see Fig. 1 Here, the P1 plane is positioned to the right of the front plane.

Figure 5 illustrates the decay behavior of the RMS pressure fluctuation data as we are traveling upstream (away from the impeller - a hydrodynamic dominated region) towards the acoustic region. The data are extracted along a line aligned with the symmetry center-line of the impeller, starting from the impeller 
hub and going towards the inlet hemispherical boundary located in the acoustic region. The observed exponential decay is characteristic to the transition from hydrodynamic to acoustic regions and has been previously exposed for turbulent 255 jets, see 35 .

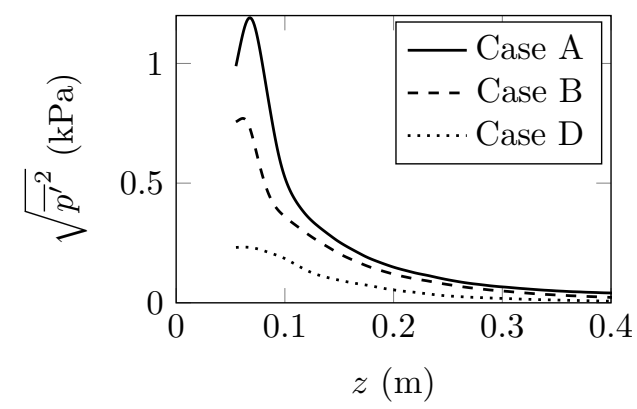

Figure 5: Root mean square of pressure fluctuation as function of distance from the impeller source along the center axis towards the acoustic near-field region.

The normalized pressure fluctuations (RMS) inside the compressor, shown for selected frequency-bands in Fig. 6, 7 and 8 , indicate the locations of specific acoustic noise production and hence, their generation mechanism. For high frequencies $\mathrm{RO}>0.6$, the highest averaged pressure fluctuations can be seen to occur in the proximity of the trailing blade tips for all operating conditions. High magnitudes occur due to flow mixing especially in the blade passages and the radial diffuser. The pressure fluctuations associated with the frequency band of $\mathrm{RO}=0.3-0.6$ distinguish the Cases $\mathrm{A}$ and $\mathrm{B}$ from Case $\mathrm{D}$. A ring-like structure of high pressure fluctuation amplitudes can be observed in the crosssectional plane located upstream of the impeller in the entrainment region for Cases A and B (visible in the inlet section and the P1 plane in Fig. 6, 7 and 8). These are the signatures of flow vortical structures circulating upstream of the impeller, which have been described in a previous study [20]. These flow features are linked with the presence of the rotating stall instability, also visible in the diffuser (at $\mathrm{RO}=0.5$ ). High amplitude fluctuations underneath the volute tongue can be noted in the band of $\mathrm{RO}=0.3-0.6$ for Case $\mathrm{B}$, while these appear more amplified in a lower frequency band, i.e. $\mathrm{RO}=0.1-0.3$, for 
Case A. The band of $\mathrm{RO}=0.1-0.3$ exhibits further high pressure fluctuation amplitudes in the blade passages. Case A can be differentiated from the other cases by the low frequency pressure fluctuations i.e. $\mathrm{RO}=0-0.1$. The flow phenomenon surge occurring at a frequency of $\mathrm{RO}=0.04(43 \mathrm{~Hz})$ is primarily responsible for the fluctuation amplitudes, which can be described as a cyclic filling and emptying process between the outlet plenum (or pipe) and the leading edge of the compressor. For a more detailed description of the flow phenomena see e.g. [19] or [20].
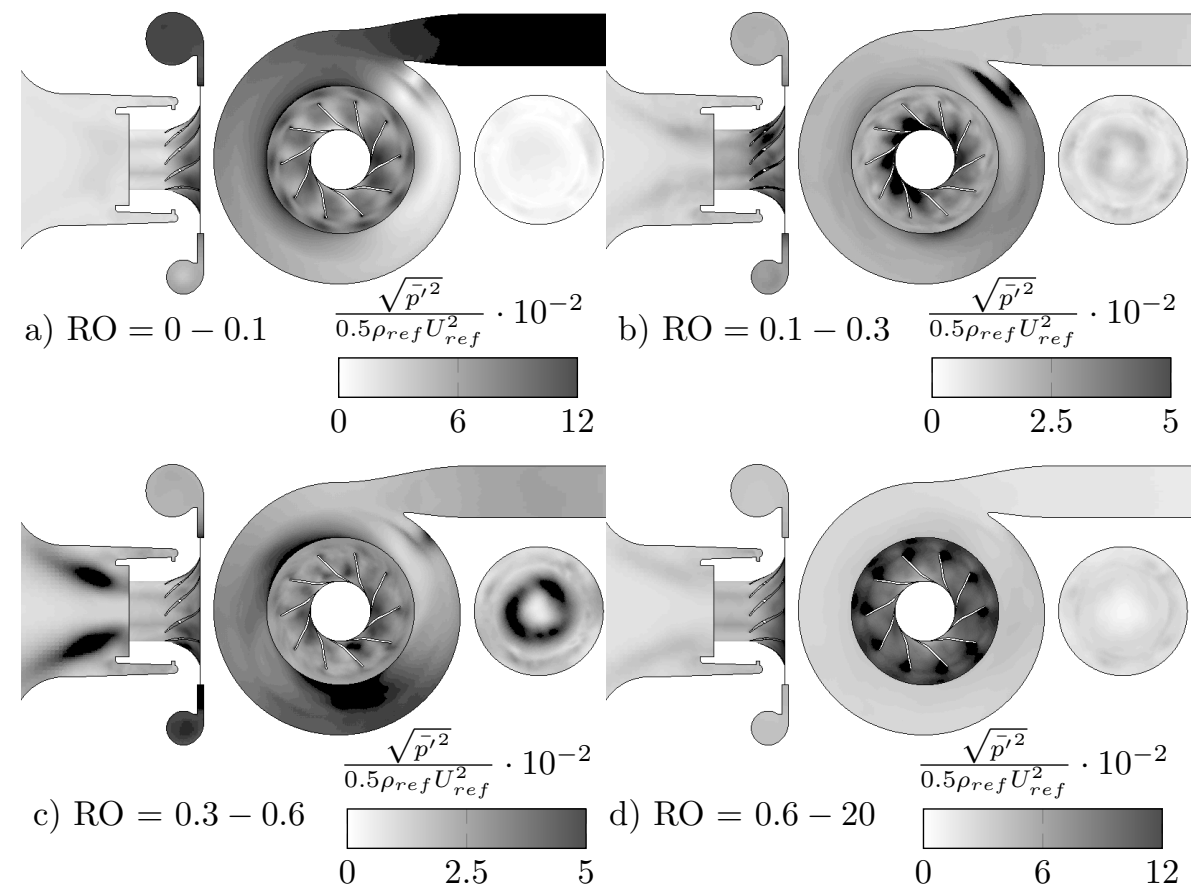

Figure 6: Normalized pressure fluctuation (root mean square - RMS) distribution for Case A. Subfigures a) to d) are around specified frequency bands. For the location of the postprocessing planes see Fig. 1. Here, the cross-sectional P1 plane upstream of the impeller is presented to the right of the front plane.

The coherence of fluctuations (static pressure and velocity) is investigated via two point space-time cross correlation analysis. The rotating stall frequency $(\mathrm{RO}=0.5)$ is clearly visible for the Cases A and $\mathrm{B}$ as shown in the space-time 


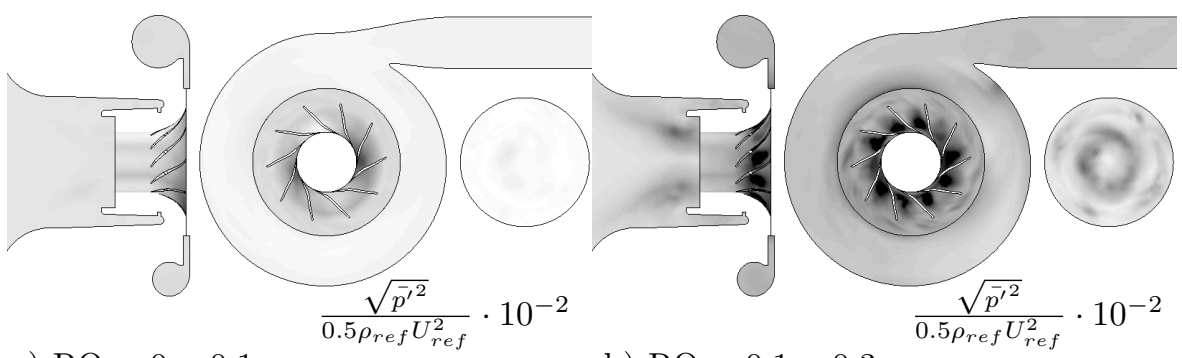

a) $\mathrm{RO}=0-0.1$

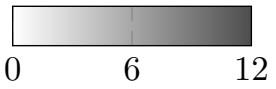

b) $\mathrm{RO}=0.1-0.3$
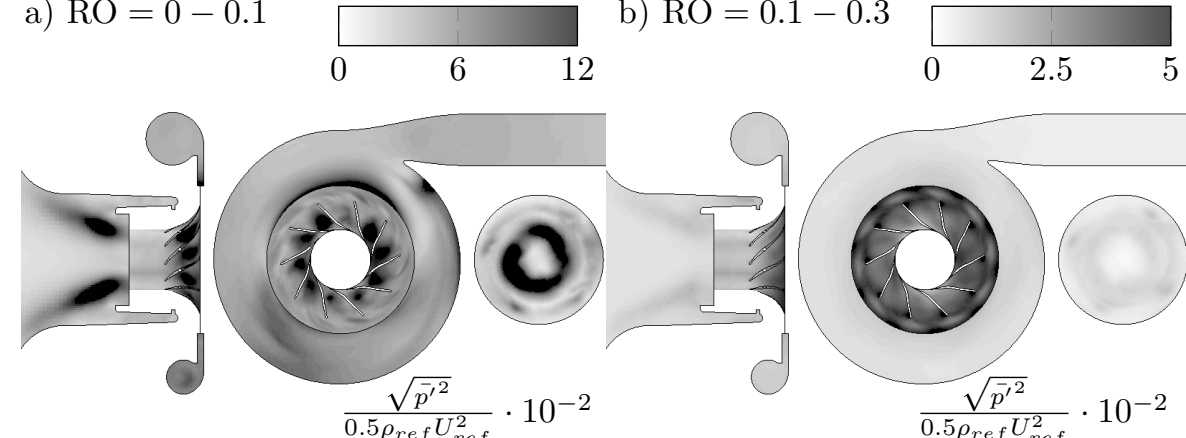

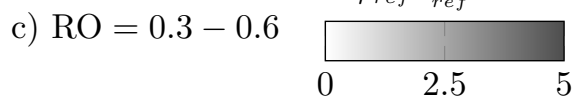

d) $\mathrm{RO}=0.6-20$

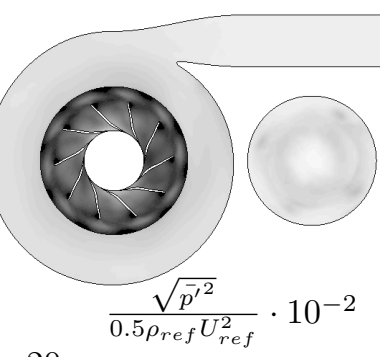

Figure 7: Normalized pressure fluctuation (RMS) distributions for Case B. Subfigures a) to d) are around specified frequency bands. For the location of the post-processing planes see Fig. 1 The cross-sectional P1 plane upstream of the impeller is presented to the right of the front plane.

correlation diagrams in Fig. 9, which are based on the pressure and axial velocity fluctuations, respectively.

The blade passing frequency can be noted in the pressure fluctuations for all cases. For Case D, the blade passing frequency is the most correlated feature. The slope $(z / t)$ of the peak pressure correlations (see Fig. 9. upper row) is the group speed for the instability propagation, which in this case is the speed of sound $(340 \mathrm{~m} / \mathrm{s})$. Moreover these disturbances based on pressure fluctuations are propagating upstream, away from the impeller region. An out-going compression wave corresponds to a negative slope in the time-space diagram. An in-going wave on the other hand would correspond to a positive slope in the time-space diagram. If there are reflections, they should manifest with a 


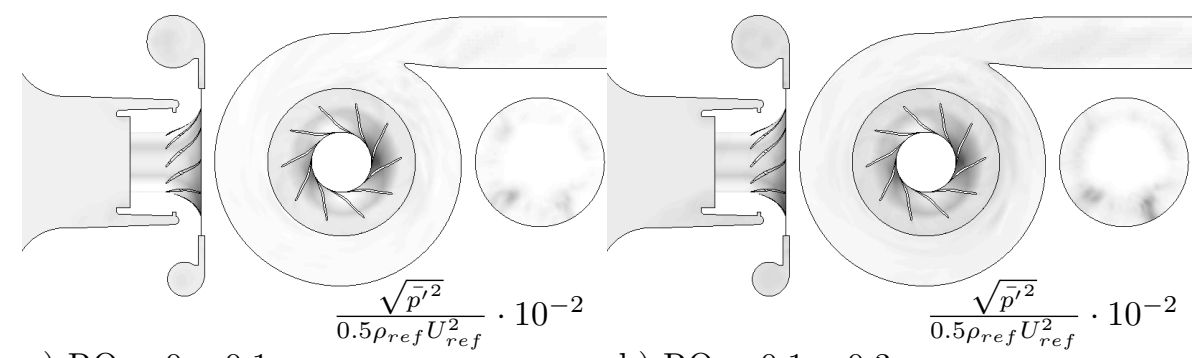

a) $\mathrm{RO}=0-0.1$

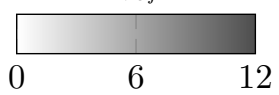

b) $\mathrm{RO}=0.1-0.3$
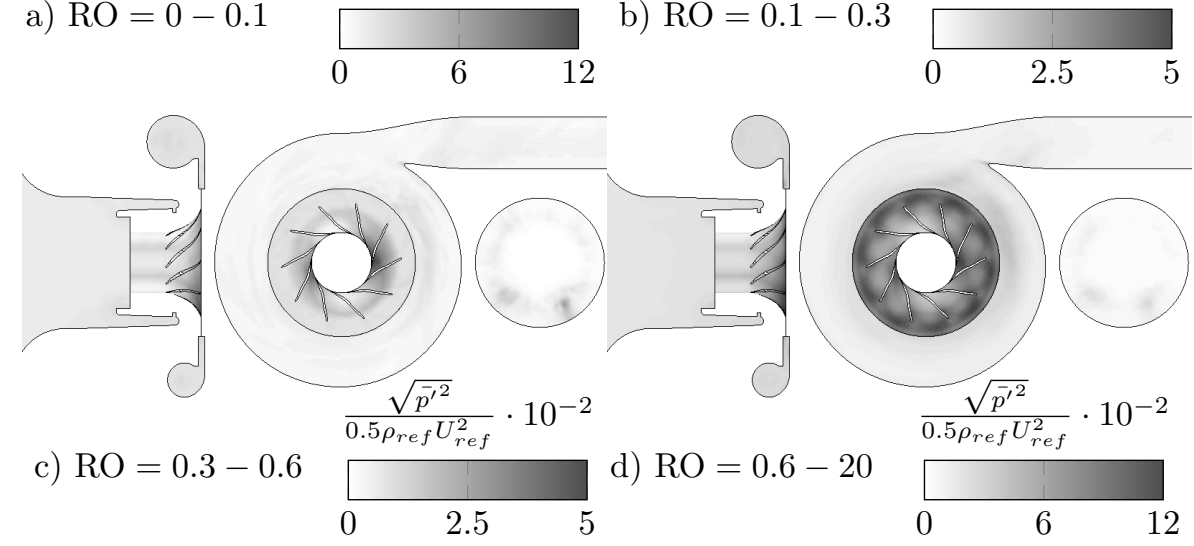

Figure 8: Normalized pressure fluctuation (RMS) distributions for Case D. Subfigures a) to d) are around specified frequency bands. For the location of the post-processing planes see Fig. 1] Here, the P1 plane is positioned to the right of the front plane.

positive slope in the time-space diagram shown in Fig. 9. Such feature with a positive slope cannot be detected. Therefore, in case of backwards reflected pressure waves their overall impact are not seen to influence the solution in the domain of interest. For the data based on axial velocity fluctuation for Cases A and B in Fig. 9, this characteristic is seen to be dominated by horizontal lines, which are identified as disturbances due to rotating stall. The space-time cross-correlation plots expose significant time-scales of dominant disturbances. Horizontal distributions can be interpreted such that disturbances appear at similar times.

A similar analysis based on cross-correlations of fluctuating pressure and tangential velocity component, respectively, carried out along a circumferential line inside of the diffuser (i.e. mid of the diffuser channel) is presented in Fig. 10 
Case A


Case B
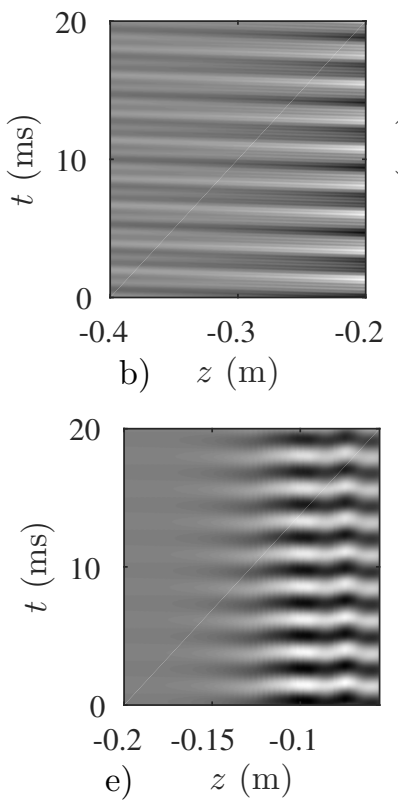

Case D
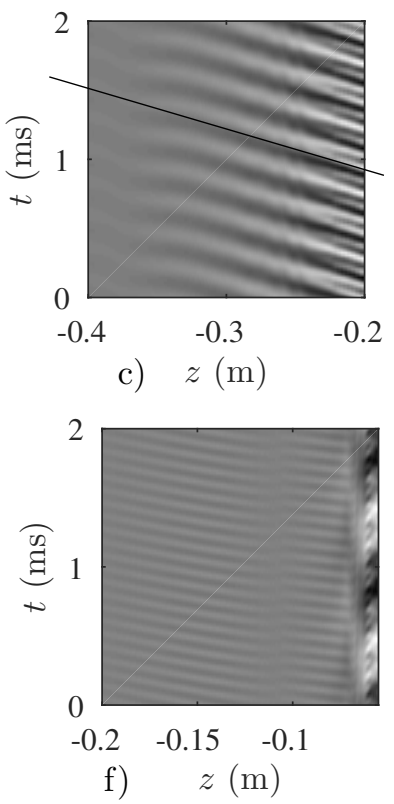

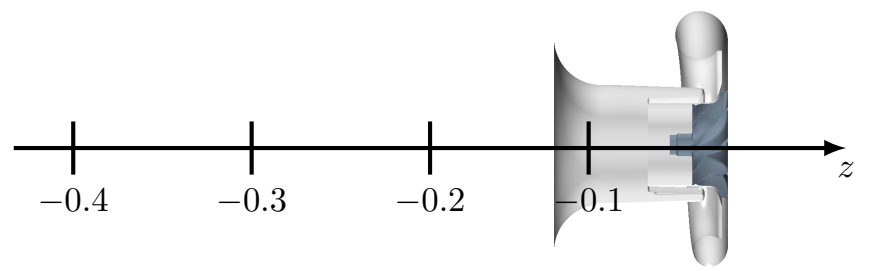

Figure 9: Space-time diagrams; normalized two point cross correlation along an upstream line. The subfigures a) to c) on the top row are based on pressure fluctuation. The subfigures d) to f) on the bottom row are based on axial velocity fluctuation. The sketch at the bottom clarifies start and end positions of the upstream line used for the cross correlation computation. The mid location along the line is used as a reference point for the correlation. The color range is from -1 to 1 . The annotation line in c) indicates the group speed slope, which coincide with the speed of sound $(340 \mathrm{~m} / \mathrm{s})$.

An interesting observation is that the momentum and pressure disturbance do not always exhibit the same time scales and hence are not always driven by the same disturbance. The data based on pressure for Cases B and D shows several disturbances or characteristic lines, one for each fluctuation disturbance associated with the blade passing. Their slopes are slightly different as com- 
pared to the upstream propagating disturbances depicted in Fig. 9, resulting in a group speed slightly larger $380 \mathrm{~m} / \mathrm{s}$ than the speed of sound at ambient conditions. Towards the off-design operating conditions, Cases A and B, the slope of the characteristics is seen to be modulated by low-frequency disturbances associates with rotating stall (note that the cross-correlations depicted in Fig. 10 are based on the circumferential velocity). Around the diffuser for Cases A and B, disturbances are seen, which correspond to crests and valleys associated with a convected rotating stall. Between the positions at 12 o'clock and under the volute tongue the rotating stall slope characteristic is dominant. Some variation is seen, which suggests that the convection speed of the rotating stall depends on the circumferential orientation. It is slightly speeding up in the region under the volute tongue and is slowing down after that. This effect may be caused by the local blockage due to the presence of the small recirculation flow region under the volute tongue. For Case A and the correlation based on pressure disturbances, the pattern shows horizontal lines. This is identified as a standing wave and thus associated with surge operation (note the different time length considered for capturing the flow frequency of surge in Case A).

A two point space-time cross correlation based on pressure disturbances is also presented along a line in the volute outlet pipe, see Fig. 11. For Cases D and B, clear characteristics due to the blade passing are seen, with group speed close to the sound speed. A standing wave is shown for Case A, associated with surge.

In order to separate the sources leading to acoustic noise generation from the hydrodynamic pressure fluctuations, the FW-H approach is employed. The sources considered with the FW-H acoustic formulation are the blade loading (dipole) term, the thickness (monopole) term, and the quadrupole term. However, the applicability of the FW-H approach in confined configurations is doubtful, c.f. [36, 30]. Therefore, the results obtained by using the FW-H approach are compared against those obtained directly from the compressible LES calculations (previously validated in 19, 20, ). The comparisons are in terms of Overall Sound Pressure Levels OASPL $[\mathrm{dB}]$ and SPL spectra obtained at the 


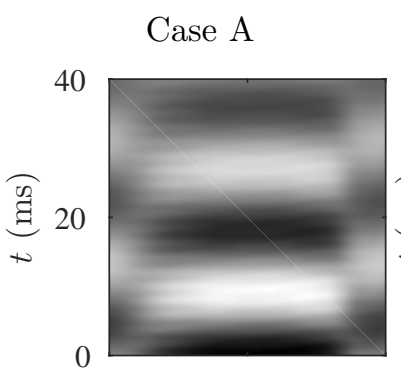

a)



Case B

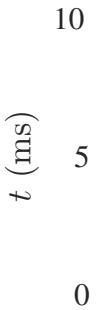

0

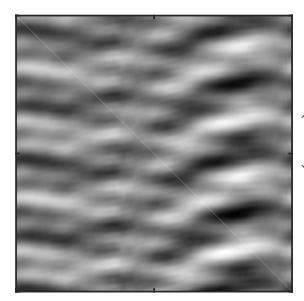

b)
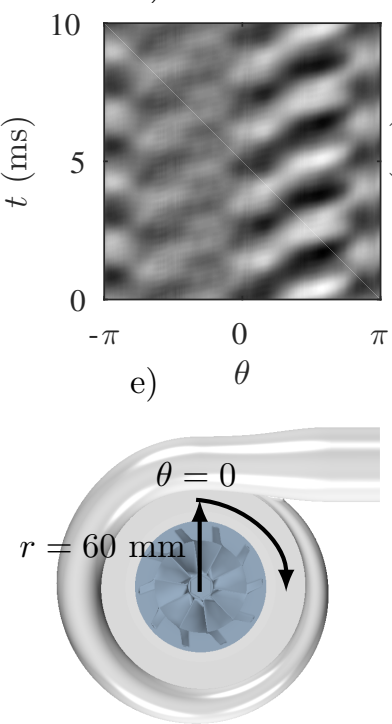

Case D

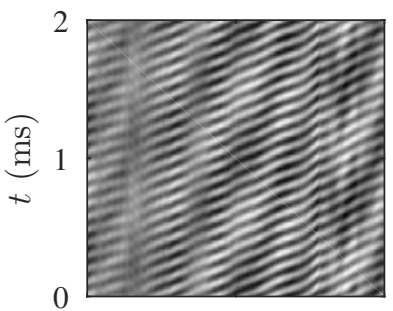

c)

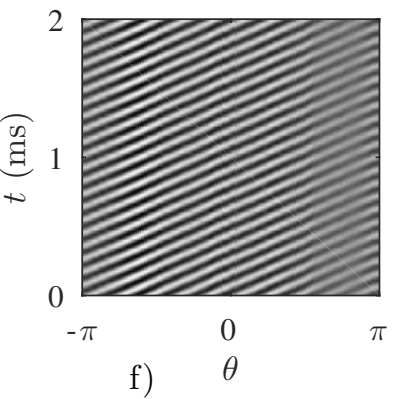

Figure 10: Normalized cross correlation along a circumferential line in the diffuser at $r=60$ $\mathrm{mm}$. Top row a), b) and c) are based on pressure. The bottom row d), e) and f) are based on tangential velocity fluctuations. The start and end points of the circumferential line as well as the reference point, are at $\theta=0$, i.e. at 12 o'clock, see sketched inset for clarification purposes. The color range is from -1 to 1 .

upstream located probe point Pin2. Table 2 shows that the maximal difference between the directly calculated OASPLs and those based on the FW-H approach is of $3 \mathrm{~dB}$. Figure 12 shows the spectra comparisons for the three different operating conditions using the direct approach and the FW-H acoustic analogy. A fair agreement (within $10 \mathrm{~dB}$ ) is found at RO frequencies below four. However, the agreement is not as good at high frequencies. This is due to the growing 
a) Case $\mathrm{A}$

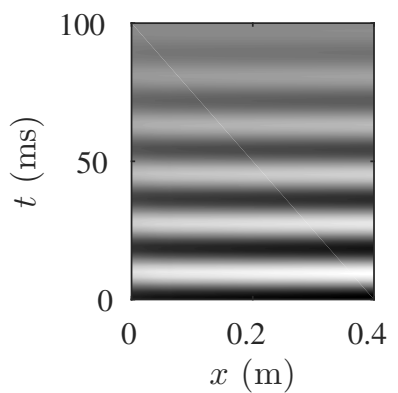

b) Case B

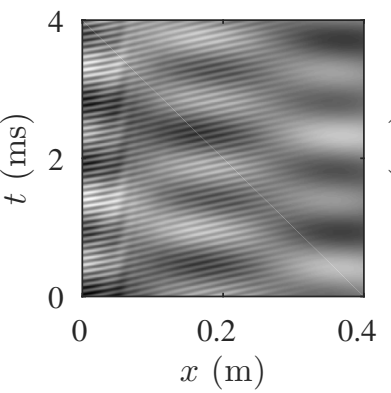

c) Case D

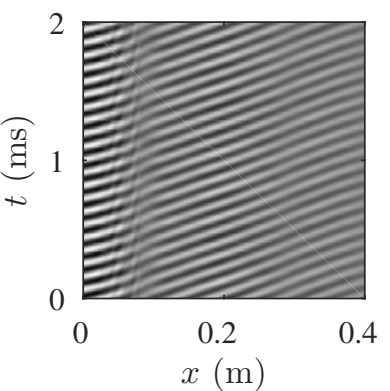

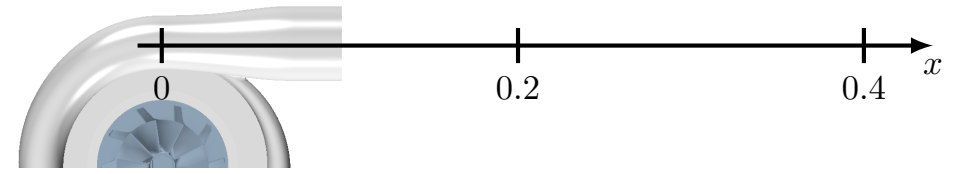

Figure 11: Normalized cross correlation based on pressure fluctuation along a line in the outlet pipe. The reference location is at $x=0 \mathrm{~mm}$. The color range is from -1 to 1 .

numerical amplitude error occurring beyond the BPF, see Fig. 2p). This causes a decay in the SPL level using the direct approach at high frequencies. One has to keep in mind that with the FW-H approach the radiated sound from the acoustic sources is propagating towards the acoustic far-field without being affected by the surrounding geometry of the compressor.

Table 2 clarifies the relative importance of the individual source terms of the FW-H approach. Note that the acoustic sources due to blade loading and blade thickness are existing on the surface of the blades, while the quadrupole acoustic source exists in the volume of the flow.

Their spatial distributions in the vicinity of the impeller are illustrated in Fig. 13. The blade loading term, $p_{L}^{\prime}$, is induced by the impeller interacting with flow structures and represents the essential source dominating the sound pressure level contribution by an order of magnitude (see Tab. 2). Especially at the leading and trailing edges, where the impeller crops up unsteady flow structures. The blade loading term reveals high amplitudes. The thickness surface term, $p_{T}^{\prime}$, establishes, in contrast to the blade loading term, towards the trailing edge of the blade suction sides where the flow separates. This term has 


\begin{tabular}{|c|c|c|c|c|c|}
\hline Case & $S P L_{L}$ & $S P L_{T}$ & $S P L_{Q}$ & $O A S P L_{F W-H}$ & $O A S P L_{\text {direct }}$ \\
\hline $\mathrm{A}$ & $132 \mathrm{~dB}$ & $24 \mathrm{~dB}$ & $78 \mathrm{~dB}$ & $132 \mathrm{~dB}$ & $130 \mathrm{~dB}$ \\
\hline $\mathrm{B}$ & $130 \mathrm{~dB}$ & $25 \mathrm{~dB}$ & $73 \mathrm{~dB}$ & $130 \mathrm{~dB}$ & $127 \mathrm{~dB}$ \\
\hline $\mathrm{D}$ & $120 \mathrm{~dB}$ & $37 \mathrm{~dB}$ & $60 \mathrm{~dB}$ & $120 \mathrm{~dB}$ & $117 \mathrm{~dB}$ \\
\hline
\end{tabular}

Table 2: Overall sound pressure level (OASPL) in (dB) at probe point Pin2 computed with FW-H and direct approach. Acoustic source contribution from blade loading $p_{L}^{\prime}$, thickness $p_{T}^{\prime}$ and quadrupole $p_{Q}^{\prime}$ terms are depicted for Case A, B and D. The overall sound pressure level (OASPL) with the FW-H is computed using: $O A S P L_{F W-H}=$ $10 \log _{10}\left(10^{\frac{S P L_{T}}{10 \mathrm{~dB}}}+10^{\frac{S P L_{Q}}{10 \mathrm{~dB}}}+10^{\frac{S P L_{L}}{10 \mathrm{~dB}}}\right) \mathrm{dB}$. The last column depicts the overall sound pressure level with the direct approach. The reference sound pressure for the $\mathrm{dB}$ scale is $20 \mu \mathrm{Pa}$
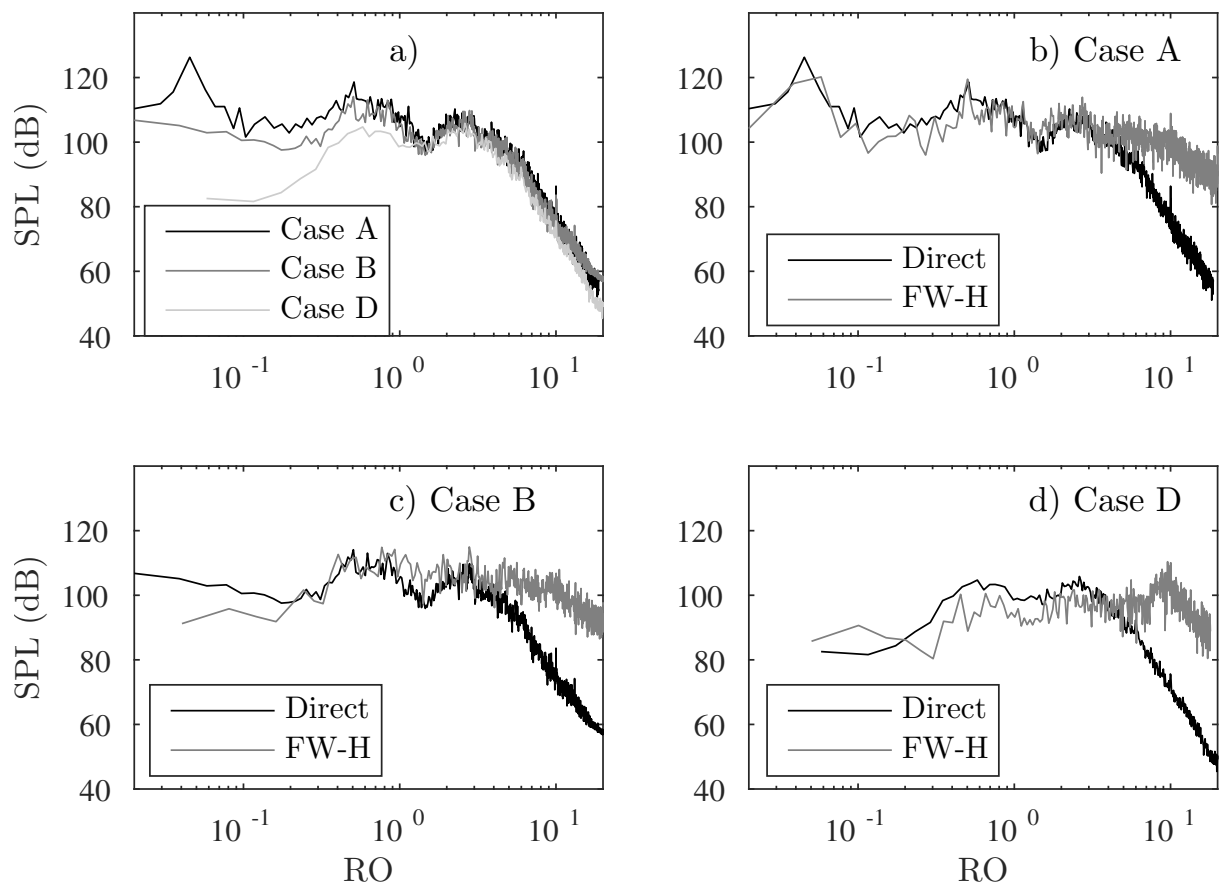

Figure 12: Sound Pressure Level in Pin2 for different flow cases, see Fig. 1 for orientation purposes. a) SPL computed with direct approach for Cases A, B and D. b) SPL computed with direct approach compared with FW-H for Case A. c) Direct approach compared with FW-H for Case B. d) Direct approach compared with FW-H for Case D. The reference sound pressure for the SPL computation in $\mathrm{dB}$ scale is $20 \mu \mathrm{Pa}$. 
the least important contribution to the overall sound pressure levels as shown in Tab. 2. The quadrupole term, $p_{Q}^{\prime}$, represents turbulent fluctuations leading to acoustic noise propagation, which is mainly generated due to separated flow from flow mixing downstream of the blades.
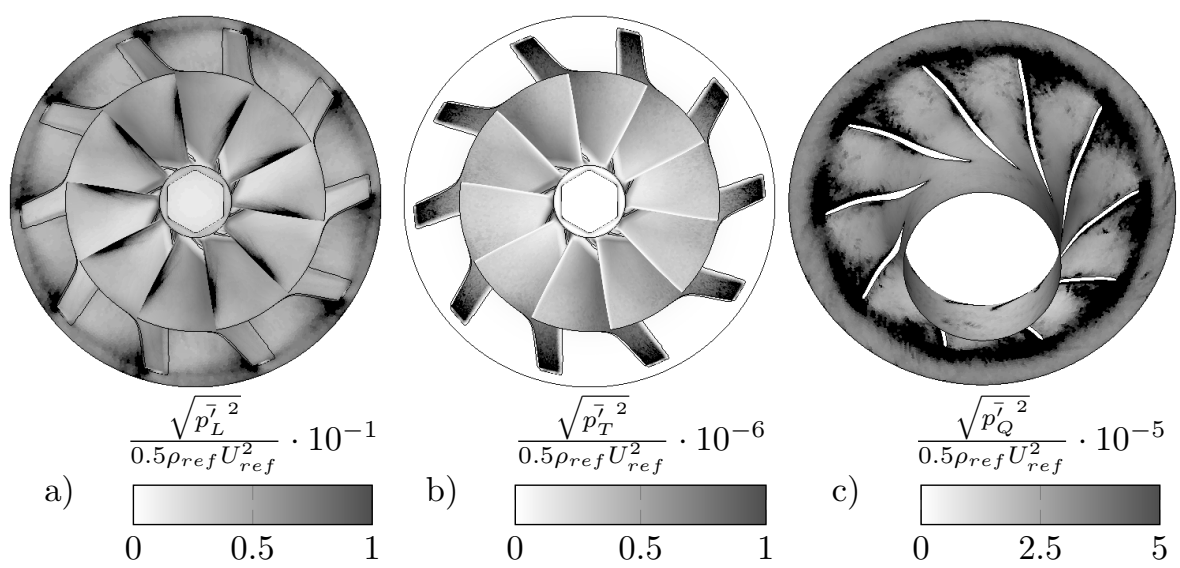

Figure 13: Acoustic intensity level contribution from a) blade loading, b) thickness, and c) quadrupole terms for Case A.

The effect of large-scale flow structures on the blade loading term can be analyzed by filtering the flow field and blade loading term at particular frequencies associated with the observed instabilities. Figure 14 shows the phase angle evolution, $\Phi$, of the reconstructed acoustic blade loading term (top row) for Case A at the low surge frequency tonality $(\mathrm{RO}=0.04)$. In this figure, correspondent flow data are included (bottom row). It shows how the coherent flow structure in surge affect the blade loading term, which in turn affects the momentum flow into the radial diffuser for one complete surge cycle. At $\Phi=3 \pi / 2$, the compressor is subjected to the filling phase of surge and streamlines illustrates downstream flow with a moderate incidence flow angle. The corresponding blade loading term depicts a neutral level. At $\Phi=0$, the streamlines depict growing incidence flow angle, which causes a building up of the blade loading level from the mid to the rear end of the impeller blades. By $\Phi=\pi / 2$ the compressor is seen to swap towards the emptying phase, and the pressure thus drops. The 
effect on the flow field is that the flow reverses mid-way in the blade passages depicting large separation zones. From the phase when the blade loading is at its minimum the flow field gradually recovers with more downstream directed flow.

Figure 15 shows the phase angle evolution, $\Phi$, of the flow perturbation (without mean flow) and the blade loading term contribution at $\mathrm{RO}=0.5$. In the P1 plane upstream of the impeller, circulating vortical structures can be observed, which have been identified as rotating stall. These vortical structures are cropped by the blades causing high amplitude contributions to the blade loading term at the leading edge. This can be observed by the high magnitude noise sources shown in Fig. 15 for all phase angles. Depending on the directionality of the vortex rotation, the flow incidence angles at the blades and hence, their loading is influenced. This causes corresponding unsteady pressures at the back wall of the impeller.

At zero phase angle, $\Phi=0$, two high-pressure zones are located at 12 and 6 o'clock near the leading edges. These correspond to the co-rotating vortex pair in the P1 plane with streamlines depicting outwards spiraling. In between, low-pressure perturbation zones can be found and they correspond to the other co-rotating vortex pair with inward spiraling. A similar distribution with two high pressure zones and two low pressure zones in between can also we seen towards the blade trailing edges at zero phase angle. A low pressure region remains between four and six o'clock at other phase angles, while the other propagates around the circumference. This asymmetry can explain the subharmonic tonality at $\mathrm{RO}=0.25$. This asymmetry is related to the asymmetric time averaged pressure field at off-design operating conditions (see e.g. [19]). In the region between 4 and 6 o'clock, the highest flow momentum is discharged into the diffuser [19, which acts as a sink for the low-pressure region. The corresponding flow field of the rotating stall can be found downstream in the diffuser and the volute regions. By a phase angle $\Phi$ of $\pi / 2$ further in the cycle, the impeller surface is rotated clockwise one quarter of a revolution. However, the pressure distribution of the rotating stall feature rotate half as much, i.e. 
$\pi / 4$, but also clockwise. In other words, in one impeller revolution, the rotating stall feature moves clockwise only half a revolution. Thus, this spinning motion circumferentially in the absolute frame explains the tonality found at $\mathrm{RO}=0.5$ in the CPSD spectra. A high pressure zone corresponds to streamlines depicting radial outgoing diffuser flow. A low pressure zone relates to reversing inward directed flow. By $\Phi=\pi$ the two high pressure zones close to the inducer are now horizontally oriented and by $\Phi=3 \pi / 2$ they are oriented diagonally between 10 and 4 o'clock. From $\Phi=3 \pi / 2$ until $\Phi=2 \pi$ the pressure and velocity distribution will gradually come back to the description stated for $\Phi=0$, and the rotating stall cycle will repeat.

At the rotating order, i.e. the angular velocity of the impeller shaft, a distinct characteristic dipole distribution is observed for all investigated cases. This is presented in Fig. 16 only for Case $\mathrm{D}$ and $\Phi=0$. The characteristic is similar with the $\mathrm{RO}=0.5$ mode described above, but exposing a distinct regularity. This means that the $\mathrm{RO}=1$ mode shape describes a circumferential rotation relative the impeller surface. The fluctuation level is particularly focused towards the exducer as well at the leading edges and the blade tips. This mode is seen to have several higher harmonics, where the RO equal 2, 3, and 4 are also presented. The dipole surface source distribution at $\mathrm{RO}=1$ including its higher harmonics are seen to correlate with an amplified SPL between $\mathrm{RO}=0.5$ and $\mathrm{RO}=1$. ${ }_{435}$ Moreover, the source distributions $\mathrm{RO}=2,3$ and 4 coincides with an amplified SPL for RO between 1.5 and 5, see Fig. 12 .

\section{Conclusions}

Aerodynamic and aero-acoustic properties in a vaneless diffuser centrifugal compressor were investigated for one constant speed-line by gradually restricting the mass flow rate going from near optimum design efficiency towards off-design surge operating conditions. The compressible flow was computed using the LES approach assuming a full annulus and grid motion of the impeller region. Assessment of flow acoustic coupling was performed using Fourier surface spec- 




a) $\Phi=0$

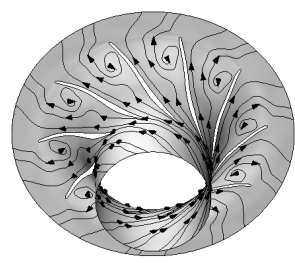

e) $\Phi=0$

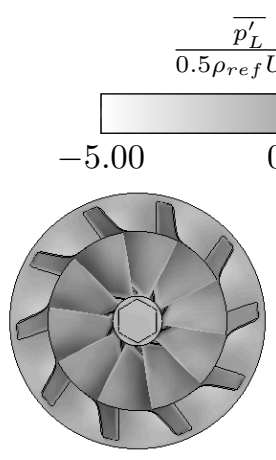

b) $\Phi=\pi / 2$

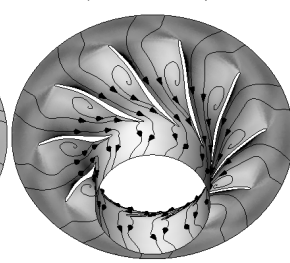

f) $\Phi=\pi / 2$

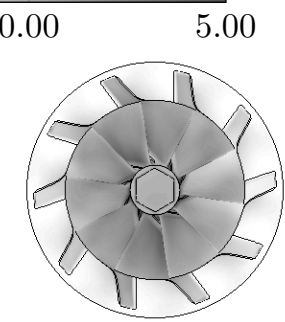

c) $\Phi=\pi$

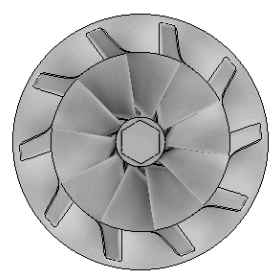

d) $\Phi=3 \pi / 2$



g) $\Phi=\pi$

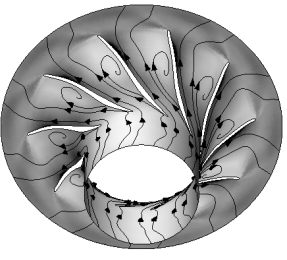

h) $\Phi=3 \pi / 2$

Figure 14: Phase evolution of acoustic blade loading term is presented on top row a), b), c) and d). Phase evolution of the modal flow perturbation is shown on the bottom row e), f), g) and $\mathrm{h}$ ). The data is for Case $\mathrm{A}$ at the surge frequency $\mathrm{RO}=0.04$. The mean velocity is not superimposed. A phase angle $\Phi=0$ represents the start of the surge cycle whereas $\Phi=\pi / 2$ indicates half way of a surge cycle.

tra and two-point cross-correlation analysis. The FW-H equation was used for classification of acoustic sources.

Computations of the unsteady flow in a centrifugal compressor at low mass flow rates revealed connections between particular flow instabilities and their acoustic signature. The mass flow rate ingested by the compressor was gradually reduced to provoke the instabilities, i.e. rotating stall and surge.

Distinct tonalities in the SPL spectra were correlated with acoustic source terms of the FW-H analogy. The blade loading source term, with dipole characteristic, was found to be several orders of magnitudes larger as compared to 


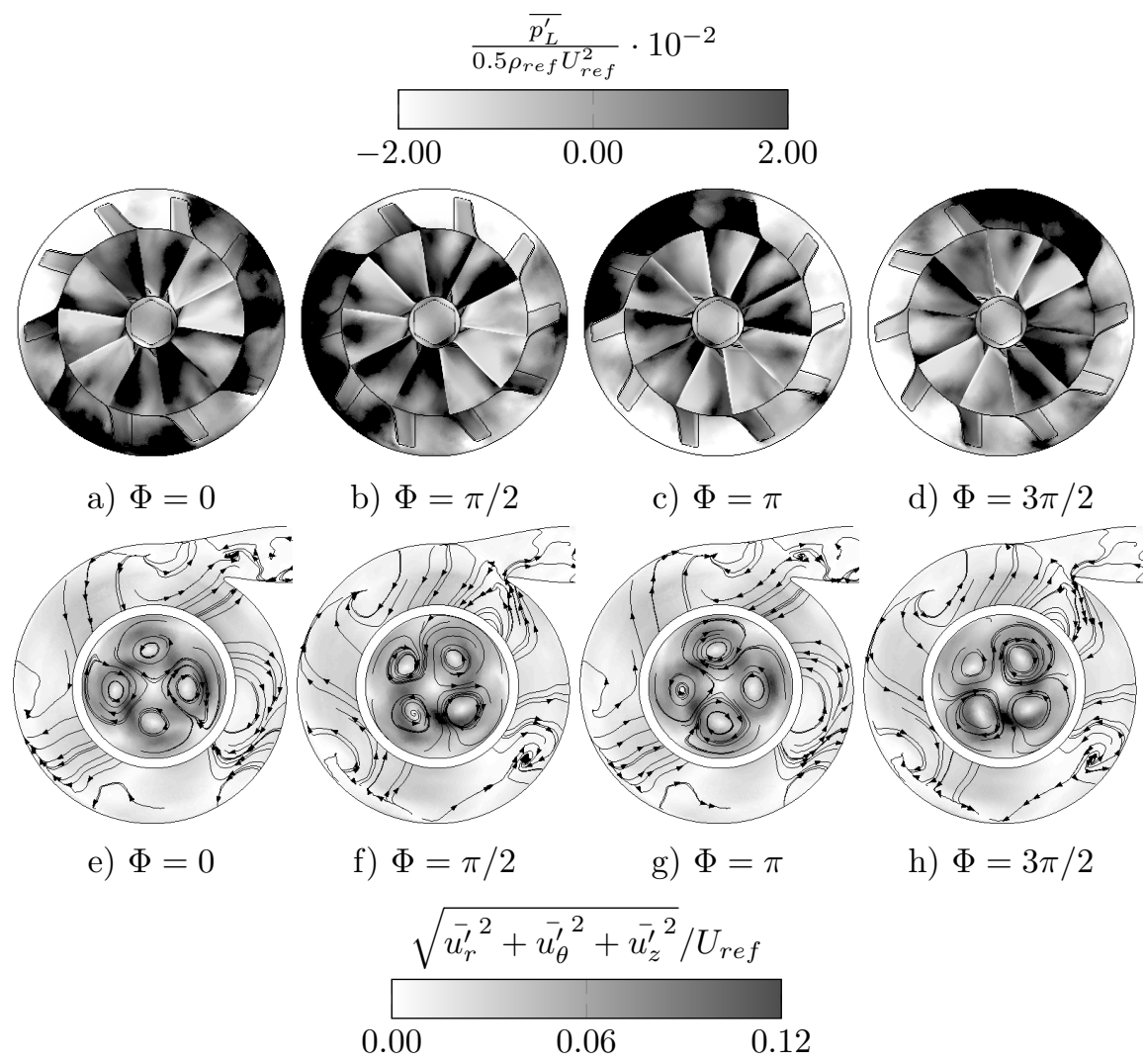

Figure 15: Phase evolution of acoustic blade loading term is presented on the top row a), b), c) and d). Phase evolution of the modal flow perturbation is shown on the bottom row e), f), $\mathrm{g}$ ) and $\mathrm{h}$ ). The data is for Case $\mathrm{B}$ at the rotating stall frequency $\mathrm{RO}=0.5$. The mean velocity is not superimposed. In the bottom row the streamlines are seeded in the front plane section and also in the P1 upstream located cross-plane, c.f. Fig. 1 for locations of post-processing planes.

thickness noise and quadrupole source terms. In fact, most noise is found to be generated at the impeller due to non-uniform blade loading. The computed acoustic power scaling does support the theoretical scaling law via Lighthill's acoustic analogy. In other words, for subsonic flow dipole sound power is stronger than the quadrupole since the scaling indicates $W_{d}: W_{q} \sim 1: M^{2}$. Moreover, monopole may only be important close to sonic tip speeds, as shown experimentally by 9 . 


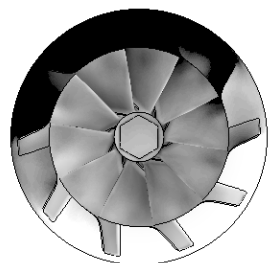

a) $\mathrm{RO}=1$

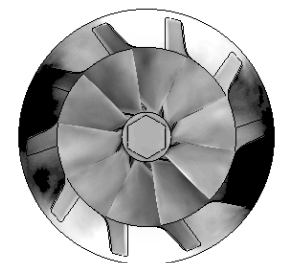

b) $\mathrm{RO}=2$

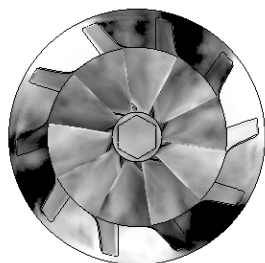

c) $\mathrm{RO}=3$

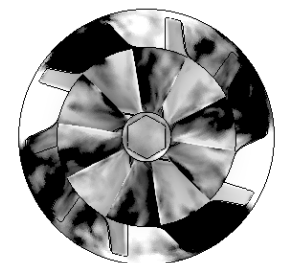

d) $\mathrm{RO}=4$

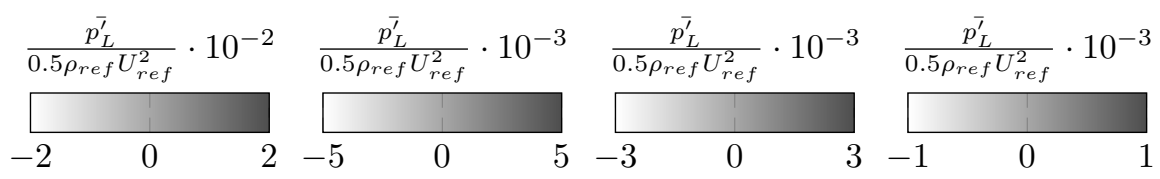

Figure 16: Front view of the impeller showing the normalized blade loading term distribution for Case D. a) At the frequency $\mathrm{RO}=1 . \mathrm{b}) \mathrm{RO}=2$. c) $\mathrm{RO}=3 . \mathrm{d}$ ) $\mathrm{RO}=4$.

For distinct tonalities found in the SPL spectra, the spatial pattern of the blade loading term on the impeller surface where investigated using modal decomposition methods. For the second most restricted mass flow rate considered (Case B), rotating stall instability was provoked. The SPL spectra include an additional tonality at $\mathrm{RO}=0.5$. In the high-frequency range $(\mathrm{RO}>2)$, the $\mathrm{SPL}$ spectra calculated for the unstable flow mass flow scenarios were found to be similar with the SPL spectrum at the stable operating condition. The RO $=0.5$ mode shape exposes a characteristic dipole distribution. The entrained vortical flow structures are chopped up at the impeller leading edge. The perturbed flow angles provoke a non-uniform pressure loading on the blades, where the high momentum flow between four and six o'clock acts as a low pressure sink. At surge operating conditions this sink becomes amplified and only one low pressure perturbation runs around the circumference at the discharge. Therefore, fluctuations of $\mathrm{RO}=0.5$ and $\mathrm{RO}=0.25$ (underneath the volute tongue) are related.

Low frequency instability at a fraction of the rotating order $\mathrm{RO}=0.04$ describing a filling and emptying process was captured at the most restricted mass flow rate case considered (Case A). This is in agreement with published literature on the subject where the mode is referred to a system instability 
named surge, see e.g. [24, 37, 11]. The SPL spectra expose a distinct tonality at this frequency and it correlates with a coherent blade loading feature distributed on the impeller surface. The acoustic sources are organized symmetrically in a circumferential arrangement with focus towards the rear part of the impeller surface as well as towards the leading edges and blade tips.

In the mid frequency range, a second amplified broadband intensity is observed in the SPL spectra between $\mathrm{RO}=2$ and four times the angular velocity of the impeller. This is in agreement with 13, 14, who discuss this feature and designate it as "whoosh noise". However, the LES data only expose a camelback shape in the far-field spectra. Such feature cannot be seen deeper inside the compressor. Therefore, it is not necessarily connected with "whoosh noise". In [38] it is mentioned that broadband noise occurs in the range $1<\mathrm{RO}<2$ for a similar size compressor as the one used in the present study. Whereas 9] relates this to "tip clearance" noise due to leakage between the shroud and the blade tips. Towards low mass flow rate operating conditions, the time-averaged pressure field becomes more asymmetric. This causes the broadband acoustic noise levels to increase by about $2-4 \mathrm{~dB}$. This manifested in static perturbation modes at all frequencies. The SPL point spectra shows narrowband features in this frequency interval, i.e. $\mathrm{RO}>1$. They corresponds to the tonality $\mathrm{RO}=1$ and higher harmonics to $\mathrm{RO}=1$. This correlates with a number of harmonic modes shapes found with modal decomposition at relevant magnitudes. The $\mathrm{RO}=2$ mode is seen to be significant in this frequency interval $(1<\mathrm{RO}<4)$.

\section{Acknowledgments}

This work was carried out within the framework of the Competence Center for Gas Exchange (CCGEx). The support from the Swedish Energy Agency (STEM), Volvo Cars, Volvo GTT, and SCANIA is greatly appreciated. The Swedish National Infrastructure for Computing (SNIC) via HPC2N and PDC, the Parallel Computing Center at KTH, are acknowledged for providing the necessary computing resources. The authors are grateful to Dr. Gutmark and 
his team at University of Cincinnati for sharing the experimental data as well as the geometry.

\section{Compliance with Ethical Standards:}

The authors declare that they have no conflict of interest.

\section{References}

[1] A. Gonzalez, M. Ferrer, M. De Diego, G. Pinero, J. Garcia-Bonito, Sound quality of low-frequency and car engine noises after active noise control, Journal of Sound and Vibration 265 (3) (2003) 663-679.

[2] A. Broatch, J. Galindo, R. Navarro, J. García-Tíscar, A. Daglish, R. Sharma, Simulations and measurements of automotive turbocharger compressor whoosh noise, Engineering Applications of Computational Fluid Mechanics (2015) 1-9.

[3] C. J. da Silveira Brizon, E. B. Medeiros, Combining subjective and objective assessments to improve acoustic comfort evaluation of motor cars, Applied Acoustics 73 (9) (2012) 913-920.

[4] L. F. Andersen, J., F. Westin, Surge definitions for radial compressors in automotive turbochargers, SAE Int. J. Engines 1 (1) (2009) 218-231. doi:https://doi.org/10.4271/2008-01-0296.

[5] R. Kabral, M. Åbom, Investigation of turbocharger compressor surge inception by means of an acoustic two-port model, Journal of Sound and Vibration 412 (2018) 270-286. doi:doi.org/10.1016/j.jsv.2017.10.003.

[6] W. Wenzel, Acoustic design for charged engines, MTZ worldwide 67 (5) (2006) 6-9.

[7] S. Fontanesi, S. Paltrinieri, G. Cantore, Cfd analysis of the acoustic behavior of a centrifugal compressor for high performance engine application, Energy Procedia 45 (2014) 759-768. 
[8] H. Rämmal, M. Åbom, Acoustics of turbochargers, SAE Technical Paper (2007-01-2205).

[9] T. Raitor, W. Neise, Sound generation in centrifugal compressors, Journal of Sound and Vibration 314 (3) (2008) 738-756.

[10] F. Kameier, W. Neise, Rotating blade flow instability as a source of noise in axial turbomachines, Journal of Sound and Vibration 203 (5) (1997) 833-853.

[11] J. Galindo, A. Tiseira, R. Navarro, M. López, Influence of tip clearance on flow behavior and noise generation of centrifugal compressors in nearsurge conditions, International Journal of Heat and Fluid Flow 52 (2015) $129-139$.

[12] F. Mendonça, O. Baris, G. Capon, Simulation of radial compressor aeroacoustics using cfd, ASME Turbo Expo 2012: Turbine Technical Conference and Exposition (GT-2012-70028).

[13] I. Tomita, S. Ibaraki, M. Furukawa, K. Yamada, The effect of tip leakage vortex for operating range enhancement of centrifugal compressor, Journal of Turbomachinery 135 (5) (2013) 051020.

[14] D. Evans, A. Ward, Minimising turbocharger whoosh noise for diesel powertrains, SAE Technical Paper (2005-01-2485).

[15] C. Teng, S. Homco, Investigation of compressor whoosh noise in automotive turbochargers, Tech. Rep. 2009-01-2053, SAE Technical Paper (2009).

555 [16] A. Karim, K. Miazgowicz, B. Lizotte, A. Zouani, Computational aeroacoustics simulation of compressor whoosh noise in automotive turbochargers, SAE Technical Paper (2013-01-1880).

[17] L. Gutin, On the sound field of a rotating airscrew, Phys. Z. Sovjetunion 9 (1936) $57-71$. 
[18] F. Farassat, Linear acoustic formulas for calculation of rotating blade noise, AIAA Journal 19 (9) (1981) 1122-1130.

[19] E. Sundström, B. Semlitsch, M. Mihaescu, Assessment of the 3d flow in a centrifugal compressor using steady-state and unsteady flow solvers, SAE Technical Paper (2014-01-2856).

[20] E. Sundström, B. Semlitsch, M. Mihaescu, Centrifugal compressor: The sound of surge, AIAA Technical Paper (AIAA2015-2674).

[21] Sound generated by turbulence and surfaces in arbitrary motion, Phil. Trans. Royal Society A264 (1969) 321-342.

[22] M. S. Howe, Acoustics of fluid-structure interactions, Cambridge Univeristy Press (https://doi.org/10.1017/CBO9780511662898).

[23] E. Guillou, M. Gancedo, E. Gutmark, A. Mohamed, Piv investigation of the flow induced by a passive surge control method in a radial compressor, Experiments in fluids 53 (3) (2012) 619-635.

[24] B. Semlitsch, M. Mihaescu, Flow phenomena leading to surge in a centrifugal compressor, J. Energy 103 (2016) 572-587.

[25] M. Giles, Non-reflecting boundary conditions for the Euler equations, Computational Fluid Dynamics Laboratory, Department of Aeronautics and Astronautics, Massachusetts Institute of Technology, 1988.

[26] A. P. Saxer, A numerical analysis of 3-d inviscid stator/rotor interactions using non-reflecting boundary conditions, Ph.D. thesis, Massachusetts Institute of Technology (1992).

[27] L. G. Margolin, W. J. Rider, A rationale for implicit turbulence modelling, International Journal for Numerical Methods in Fluids 39 (9) (2002) 821841. 
[28] C. Fureby, F. F. Grinstein, Large eddy simulation of high-reynolds-number free and wall-bounded flows, Journal of Computational Physics 181 (1) (2002) 68-97.

[29] J. H. Ferziger, M. Peric, Computational methods for fluid dynamics, Springer Science \& Business Media, 2012.

[30] F. Farassat, S. Padula, M. Dunn, Advanced turboprop noise prediction based on recent theoretical results, Journal of Sound and Vibration 119 (1) (1987) 53-79.

[31] K. S. Brentner, F. Farassat, Analytical comparison of the acoustic analogy and kirchhoff formulation for moving surfaces, AIAA journal 36 (8) (1998) $1379-1386$.

[32] D. Casalino, An advanced time approach for acoustic analogy predictions, Journal of Sound and Vibration 261 (4) (2003) 583-612.

[33] E. Sundström, B. Semlitsch, M. Mihaescu, Generation mechanisms of rotating stall and surge in centrifugal compressors, Flow, Turbulence and Combustiondoi:doi.org/10.1007/s10494-017-9877-z.

[34] M. Shur, M. Strelets, A. Travin, J. Christophe, K. Kucukcoskun, C. Schram, S. Sack, M. Åbom, Effect of inlet distortions on ducted fan noise, AIAA technical paper (AIAA2016-2819).

[35] T. Suzuki, T. Colonius, Relation between instability waves and lowfrequency jet noise investigated with phased-microphone arrays, AIAA technical paper (AIAA2006-0622).

[36] M. Åbom, An introduction to flow acoustics, TRITA-AVE 2006:04, ISSN 1651-7660, ISRN/KTH/AVE/N-06/04-SE.

[37] B. Semlitsch, V. Jyothishkumar, M. Mihaescu, L. Fuchs, E. J. Gutmark, Investigation of the surge phenomena in a centrifugal compressor using 
large eddy simulation, in: ASME 2013 International Mechanical Engineering Congress and Exposition, Vol. IMECE2013-66301, American Society of Mechanical Engineers, 2013, pp. V07AT08A053-V07AT08A053.

[38] A. J. Torregrosa, A. Broatch, R. Navarro, J. García-Tíscar, Acoustic characterization of automotive turbocompressors, International Journal of Engine Research (2014) 1468087414562866. 